\section{No place like exosome}

\section{By Kai-Jye Lou, Staff Writer}

University of Oxford researchers have generated targeted exosomes that can deliver RNAi payloads across the blood brain barrier, which could simplify the delivery of oligonucleotide-based therapeutics into the CNS. ${ }^{1}$ The researchers are now refining the methods they use to load and target the exosomes.

Exosomes are vesicles secreted by cells that facilitate intercellular communication and removal of unneeded cellular components. A 2007 study from a Swedish group showed that exosomes also can shuttle RNA. ${ }^{2}$

"Our group found that exosomes could efficiently deliver RNA from cell to cell and modify the activity of the recipient cell," said Jan Lötvall, principal investigator on the study and a professor of clinical allergy at the University of Gothenburg. "This intrinsic ability could make exosomes ideal as vectors for delivering RNA to target cells."

Now, an Oxford team led by Matthew Wood has built upon the Gothenburg group's findings. "We thought that we could exploit this natural system to deliver therapeutic RNAs" to specific cell types via systemic injection, said Wood, university lecturer in the Department of Physiology, Anatomy and Genetics at Oxford. "The two major hurdles addressed in our current study are how to target these exosomes to the desired cell type and how to insert a relevant drug or RNA molecule into an exosome."

To address the first hurdle, the Oxford researchers used immature mouse dendritic cells (DCs) as the exosome source and engineered the DCs to express lysosomal-associated membrane protein 2 (Lamp2; Lamp-2b) fused with the neuron-targeting rabies viral glycoprotein (RVG) peptide. For the second, the group used electroporation to insert a small interfering RNA payload into a purified sample of targeted exosomes.

In two proof-of-concept studies in wild-type mice, tail vein injection with targeted exosomes containing siRNA directed against a housekeeping gene or $\beta$-site APP-cleaving enzyme 1 (BACE1) resulted in neuron-specific knockdown of mRNA and proteins encoded by the target genes compared with no treatment.

BACE1 is a therapeutic target for Alzheimer's disease (AD).

Results were published in Nature Biotechnology.
"Other groups have certainly attempted to use synthetic nanoparticles to deliver oligonucleotides across the blood brain barrier, but thus far the data have not been very convincing," said Wood. "With our approach, we were able to achieve $60 \%-70 \%$ knockdown of the targeted genes in the brain, which is very good. We did compare our targeted exosomes with a previously reported method for delivering siRNA across the blood brain barrier ${ }^{3}$ and found that our approach achieved about twice the knockdown efficiency using only $10 \%$ of the siRNA."

Wood added that his group did not detect signs of immunogenicity or see any adverse events in the mice. He noted that the team used exosomes from immature DCs, which he said may be less immunogenic than mature DCs.

"These initial data suggest that targeted exosomes are of potential value for delivering RNAi-based therapeutics across the blood brain barrier to cells of the CNS," said Dinah Sah, VP of research, CNS and oncology at Alnylam Pharmaceuticals Inc. "Since exosomes are naturally occurring particles, they may be particularly efficient and also well tolerated for delivery of payloads, such as siRNAs."

"One of the main challenges with developing drugs for CNS diseases has been breaching the blood brain barrier," said John Rossi, chair and professor of the department of molecular biology at the Beckman Research Institute at City of Hope. "Direct injection is the current approach for delivering oligonucleotide-based therapeutics across the blood brain barrier, which does have risks" including infection in addition to hazards that stem from the need to remove a piece of the skull to access parts of the brain.

Rossi said those risks may be acceptable "for serious neurodegenerative diseases like Huntington's. However, the risks associated with direct injection may make it less suitable for something like early stage Alzheimer's."

Sah said it would be important to show that the results in the paper translate both to additional targets in cells within the CNS and from rodent models to nonhuman primates. She added that it would be important to define the therapeutic index of siRNA-loaded exosomes and carry out additional studies to evaluate their immunogenicity and specificity.

Rossi also said the researchers should evaluate whether the targeted exosomes facilitate the movement of potentially undesirable molecules into the CNS, for example, by carrying such molecules inside the exosome in addition to therapeutic payload or by allowing such molecules to cross the blood brain barrier as the exosome passes through.

Alnylam's most advanced program, ALN-RSV01, is a respiratory syncytial virus (RSV)-specific siRNA in Phase II testing. In the neurology space, the company's ALN-HTT is in preclinical development. This siRNA against huntingtin (HTT) is delivered to the CNS via an implantable infusion system from Medtronic Inc. 


\section{ANALYSIS}

\section{Production value}

In addition to exploring the types of CNS cells amenable to exosome delivery, another outstanding issue includes the scalability of the approach.

"Production and manufacturing is going to be a major question with the exosome approach," Rossi told SciBX. "It will be important to determine the composition of these exosomes and to know whether one can make enough of the product to deliver an effective dose to patients and whether the consistency of the product can be maintained."

"If we knew what the components of the exosome were, we could conceivably create synthetic molecules that mimic their function," said Rossi.

Lötvall added it would be important to consider whether it is better to mass produce the exosomes in vitro and turn them into off-the-shelf products or to obtain the exosomes directly from the patient and then process and load them with the RNA cargo. He noted that the former could have higher safety and regulatory hurdles, whereas the latter may have supply limitations and be a more labor-intensive process that would have fewer toxicological concerns.

Wood said his group is trying to improve its exosome delivery method in three areas. "The first area of improvement would be to increase the efficiency at which we are able to load siRNA and other cargos into the exosomes, which could further improve our knockdown efficiency," he told SciBX. "The second area we're working on is in improving the methods we use to target the exosomes to a particular cell type, and the third area is to identify the best cells from which to derive these exosomes. While it remains to be proven, it may be better to derive exosomes from neurons if we are intending to deliver exosomes to neurons. But unlike dendritic cells, neurons are not a very rich source of exosomes."

The University of Oxford has two pending patents covering methods for loading and targeting exosomes to various tissues. The work is available for licensing from Isis Innovation Ltd., the university's technology transfer arm.

Lou, K.-J. SciBX 4(14); doi:10.1038/scibx.2011.386

Published online April 7, 2011

\section{REFERENCES}

1. Alvarez-Erviti, L. et al. Nat. Biotechnol.; published online March 20, 2011; doi:10.1038/nbt.1807

Contact: Matthew J.A. Wood, University of Oxford, Oxford, U.K. e-mail: matthew.wood@dpag.ox.ac.uk

2. Valadi, H. et al. Nat. Cell Biol. 9, 654-659 (2007)

3. Kumar, P. et al. Nature 448, 39-43 (2007)

COMPANIES AND INSTITUTIONS MENTIONED

Alnylam Pharmaceuticals Inc. (NASDAQ:ALNY), Cambridge, Mass. Beckman Research Institute at City of Hope, Duarte, Calif. Isis Innovation Ltd., Oxford, U.K.

Medtronic Inc. (NYSE:MDT), Minneapolis, Minn.

University of Gothenburg, Gothenburg, Sweden University of Oxford, Oxford, U.K. 